\title{
Thermal stability of self-gravitating, optically thin accretion disks
}

\author{
G. Bertin and G. Lodato \\ Scuola Normale Superiore, Piazza dei Cavalieri 7, 56126 Pisa, Italy \\ Received 20 July 2000 / Accepted 7 February 2001

\begin{abstract}
In the dynamics of accretion disks, the presence of collective effects associated with the self-gravity of the disk is expected to affect not only the momentum transport, but also the relevant energy balance equations, which could differ substantially from the non-self-gravitating case. Here we follow the model that, when the disk is sufficiently cold, the stirring due to Jeans-related instabilities acts as a source of effective heating. The corresponding reformulation of the energy equations allows us to: $(i)$ demonstrate how self-regulation can be established, so that the stability parameter $Q$ is maintained close to a threshold value, with weak dependence on radius; (ii) rediscuss the opacity properties in the self-gravitating regime. In particular, we show that, if cooling is dominated by bremsstrahlung, an optically thin stationary accretion solution is thermally stable, even in the non-advective case, provided the disk is self-gravitating. The details of the cooling function have little effect on the structure of such accretion disk, which is in any case induced by self-gravity to self-regulate. This condition of self-gravitating accretion is expected to be appropriate for the outer regions of many disks of astrophysical interest. With the reformulation of the energy equations described in this paper, we have also secured: (iii) a starting point for the study of the emission properties of self-gravitating accretion disks; $(i v)$ a tool to analyze the structure of the transition region, where the disk becomes self-gravitating.
\end{abstract}

Key words. accretion, accretion disks - gravitation - hydrodynamics - instabilities

\section{Introduction}

A key step in the modeling of accretion disks is the detailed description of the balance between heating and cooling terms in the energy transport equations (see Pringle 1981). In this context, two hypotheses have been generally considered. The first states that the only source of heating in the disk is viscous dissipation, so that the available power is provided by the release of gravitational binding energy from the infalling matter. The second hypothesis is that cooling is essentially radiative. This latter hypothesis leads to different disk models, depending on whether the disk is optically thick (as in the "standard" model of Shakura \& Sunyaev 1973) or optically thin (as in the model of Shapiro et al. 1976; hereafter SLE). A more recent development in the description of the energetics of accretion disks has been the recognition that radiative cooling may sometimes be insufficient. Under these circumstances, most of the energy remains stored in the disk rather than being radiated away, leading to the so-called advection-dominated accretion flows (ADAF; Narayan \& Yi 1994). This situation is described by a new equation of energy transport relevant to the radial direction including an advection term (see Sect. 2 below).

Send offprint requests to: G. Lodato,

e-mail: lodato@cibs.sns.it
Once the general characteristics of stationary models are established, one can proceed to address the problem of their stability. Different types of instabilities have been considered, leading to important conclusions about the possibility of applying the models to concrete systems. In one line of research, the stability analysis is carried out with the goal of identifying microscopic collective mechanisms able to justify the viscosity $\alpha$-prescription that is commonly introduced, following Shakura \& Sunyaev (1973), in order to bypass the low level of viscosity predicted by classical arguments. Other interesting stability aspects have also been investigated. In particular, the thermal-viscous instability related to hydrogen ionization (Meyer \& Meyer-Hofmeister 1981) has been invoked to explain the variability of dwarf novae. In contrast, the optically thin SLE model has been commonly regarded as inapplicable, because of the thermal instability that is found to occur when optically thin bremsstrahlung is the dominant cooling mechanism. The thermal stability properties of the models depend strongly on the assumptions about the balance between heating and cooling terms (for example, it is well known that, in the ADAF context, optically thin solutions exist in the inner disks and are thermally stable; see Sect. 2 and Narayan \& Yi 1995). Indeed, it has been noted (Piran 1978) that small variations in the 
viscosity law can change the stability of accretion disk models drastically.

The self-gravity of the disk can change this picture significantly. It may have an obvious impact on the radial and on the vertical force relations (Paczyński 1978). Furthermore, it has long been recognized that non-axisymmetric instabilities associated with the selfgravity of the disk can affect the properties of angular momentum transport and hence the relevant viscosity law (e.g., see Lin \& Pringle 1990; Laughlin \& Bodenheimer 1994), although it still remains to be clarified whether such modifications can be reconciled with the standard $\alpha$-prescription. Recently we have argued (Bertin 1997; Bertin \& Lodato 1999) that self-gravity should be properly incorporated also in the energy balance equations, which should thus take a more general form than usually considered. In these first investigations we actually replaced the energy equations by a self-regulation prescription demanding that the axisymmetric stability parameter $Q$ is maintained close to a value $\bar{Q}$, basically dictated by the condition of marginal Jeans stability. The physical justification for the existence of an efficient dynamical thermostat of this kind is based on the presence of suitable competing heating and cooling mechanisms, but was not made explicit in terms of a set of consistent equations for the energy transport. This simplified approach is adequate for the construction of dynamical models, and has already led to some unexpected results (in particular, the natural occurrence of flat rotation curves). Yet, it deliberately avoids the issue of the predicted emission of the accretion disk, which has played an important role in the comparison of models with observations in this general research area. We anticipate that the disk self-gravity should be significant only in the cold outer disk. In this respect, the identification of a consistent set of energy equations would also be desirable in order to set up a proper matching of solutions across the relevant transition region.

In this paper, we include the effect of Jeans-related instabilities in the energy balance equations by means of a term of effective heating. Such a heating term is modeled as a function steeply rising when $Q$ falls below a threshold value $\bar{Q}$ and practically vanishing when $Q$ rises above $\bar{Q}$. We then show how this more general energy equation leads to the self-gravitating, self-regulated accretion disk solution, characterized by a value of $Q$ very close to $\bar{Q}$ and only weakly dependent on radius. This stationary solution, although non-advective and optically thin, turns out to be thermally stable.

The paper is organized as follows. In Sect. 2 we briefly summarize the arguments that lead to the balance of heating and cooling terms in current theories of accretion disks. In Sect. 3 we propose a way to incorporate the effects of self-gravity in the energy equations by means of an additional heating term. In Sect. 4 we then provide and discuss a complete set of equations for the construction of the full models of self-gravitating accretion disks. In Sect. 5 we outline the structure of the stationary models and study their thermal stability. The conclusions of the paper are given in Sect. 6, where we briefly comment on possible astrophysical applications.

\section{Energy balance in non-self-gravitating disks}

A generally accepted approach to the construction of accretion disk models starts from the assumption that the disk is geometrically thin (so that vertically-integrated and vertically-averaged quantities can be usefully introduced) and adopts a fluid model, specified by the continuity equation, the radial component of the Euler equation, the conservation of angular momentum in the presence of viscous torques, a viscosity prescription (such as the $\alpha$-prescription), an expression for the vertical thickness of the disk (to be derived from the solution of the vertical hydrostatic equilibrium equations), and the relevant energy equations. In the following, we will refer to vertically integrated or averaged quantities, such as the surface density $\sigma$, as "surface" quantities, while we will refer to other quantities, such as the volume density $\rho$, as "volume" quantities.

The energy equations are generally based on two hypotheses, of purely viscous heating (with the addition, in some cases, of irradiation from the inner disk or from the central object) and of purely radiative cooling. The first hypothesis is thus contained in an expression for the heating rate $Q^{+}$in terms of "surface quantities":

$Q^{+}=\nu \sigma\left(r \Omega^{\prime}\right)^{2} \equiv D(r)$

where $\nu$ is the viscosity coefficient and $\Omega=\Omega(r)$ is the differential rotation (usually taken to be Keplerian).

The second hypothesis, that cooling is entirely radiative, implies that the cooling rate per unit surface $Q^{-}$can be calculated from the "volume" properties of the disk and the assumed radiation mechanisms:

$Q^{-}=R$.

The energy equations are then split into an equation for the radial transport and an equation for the vertical transport. In practice, in systems with given physical properties, one should carefully check the validity of this separation, which is natural only in the limit of thin disks. For the horizontal transport the equation is the so-called "advection" equation:

$\sigma u T_{0} \frac{\mathrm{d} s}{\mathrm{~d} r}=Q^{+}-Q^{-}$

where $u$ is the radial velocity, $T_{0}$ is the midplane temperature, and $s$ is the entropy per unit mass. This equation states that, locally, the unbalance between energy gained from heating and energy lost from cooling is stored in the disk and transported radially along with the infalling matter. Entropy in Eq. (3) is calculated based on the first principle of thermodynamics:

$T \mathrm{~d} s_{\mathrm{v}}=\mathrm{d} Q=c_{\mathrm{v}} \mathrm{d} T+p \mathrm{~d}(1 / \rho)$

where $\rho$ is the volume density, $p$ is the pressure, $c_{\mathrm{v}}$ is the specific heat at constant volume and $s_{\mathrm{v}}$ is the "volume" 
entropy. After averaging over the vertical direction one defines the averaged "surface" entropy so that:

$T_{0} \frac{\mathrm{d} s}{\mathrm{~d} r}=\frac{1}{\gamma-1} \frac{\mathrm{d} c^{2}}{\mathrm{~d} r}-\frac{c^{2}}{\rho_{0}} \frac{\mathrm{d} \rho_{0}}{\mathrm{~d} r}$,

where $c$ is the thermal speed on the equatorial plane, $\rho_{0}$ is the central volume density, so that $\sigma=2 \rho_{0} h$ ( $h$ is the geometrical thickness) and $\gamma$ is the specific heat ratio. Note that in Eq. (3) one neglects the effects of horizontal radiative energy transport (but see Eq. (3) in Narayan \& Popham 1993). For the vertical transport one uses the equations of radiative transport, which, integrated over the $z$-coordinate, give rise to Eq. (2).

A final remark is in order. The above equations are a set of differential equations, and hence they should be supplemented by a set of appropriate boundary conditions. However, for standard disks (see Sect. 2.1 below), in which one neglects advection terms, the system reduces to a set of algebraic equations and no boundary conditions are thus required. In contrast, ADAF solutions are based on the full differential problem. Even in this case, however, the self-similar solution found by Narayan \& Yi (1994) (see Sect. 2.2) avoids the need for specifying the boundary conditions. Other ADAF models take such a self-similar solution as the approximate solution to be matched at large radii. In this context, it has been noted (Yuan 1999) that the boundary conditions are, indeed, important in determining the structure of the accretion disk and should be handled carefully.

\subsection{Standard disk}

The so-called standard disk solutions are those for which, in Eq. (3), the dominant terms are $Q^{+}$and $Q^{-}$, so that the advection equation reduces to:

$Q^{+} \approx Q^{-}$.

If we express this relation in view of the physical hypotheses mentioned previously, then the energy balance equation for standard disks is:

$D=R$.

As $R$ describes the emission of radiation from the disk, Eq. (7) shows that the emission is related to $D$ in a simple way, largely independent of the detailed construction of the model. On the other hand, Eq. (7), involving all the surface quantities $\left(\sigma, T_{0}, \ldots\right)$, is the closure relation needed to calculate the structure of the accretion model.

\subsection{ADAF models}

In some cases, the output of radiation may be insufficient so that the balance between $Q^{+}$and $Q^{-}$in Eq. (3) is not possible. In this more general case, one cannot use Eq. (6) (and hence Eq. (7)). This situation has often been described in terms of a convenient parameter, called $f$, defined as:

$f=1-\frac{R}{D}$ so that Eq. (3) can be rewritten as:

$\sigma u T_{0} \frac{\mathrm{d} s}{\mathrm{~d} r}=f D$

For specified radiation processes, it is clear that Eqs. (8) and (9) together can close the model, which is characterized (for given values of the viscosity parameter $\alpha$ and of the mass and angular momentum accretion rates $\dot{M}, \dot{J})$ by a profile $f=f(r)$ determined by Eq. (8). In reality, it has been noted that, at a given radius $r$, by considering the relation between $f$ and $\dot{M}$ ( $\alpha$ and $\dot{J}$ being fixed), there are in general three branches of solutions (at least for sufficiently small values of $\dot{M})$ : $(i)$ one branch corresponding to $f \ll 1$, so that (see Eq. (8)) $R \approx D$, is similar to the solution of standard disks, (ii) one new branch, called ADAF, with $f \approx 1$, is characterized by the balance between viscous heating and the advection term (see Eq. (9)); for this branch there is little emission of radiation, as $R=(1-f) D \ll D$. Finally, a third branch (iii), for intermediate values of $f$, is thermally unstable and corresponds to the SLE solution.

Treating $f$ as a given parameter, Narayan \& Yi (1994) have shown that models closed only by Eq. (9) (that is, not taking into account the radiation properties) admit a simple, self-similar solution. This solution can be seen as a first approximation to the ADAF solution on a wide (but, in any case, limited) radial range.

\section{Influence of self-gravity on the energy balance}

From the solution of the non-self-gravitating disk equations, it is interesting to check the behavior of the parameter $Q$, which, as is well known, regulates the stability of the disk against axisymmetric Jeans instability. For a fluid one-component disk, the parameter $Q$ is defined as:

$Q=\frac{c \kappa}{\pi G \sigma}$

where $\kappa$ is the epicyclic frequency (equal to $\Omega$ in the Keplerian case). Self-gravity becomes important at large radii. In fact, $Q(r)$ is expected to be a decreasing function of radius. For example, for the optically thick disk dominated by the Kramers opacity (the outer region of the Shakura \& Sunyaev 1973 disk), it is proportional to $r^{-9 / 8}$. When the standard model is applied to concrete cases (for example, the conditions typical of an AGN) $Q$ is found to plunge well below unity in regions of interest (Kumar 1999). This behavior is untenable. It is only a reminder that the equations considered initially to construct the accretion disk model must be reformulated so as to incorporate the important role of the disk self-gravity (e.g., see Kolykhalov \& Sunyaev 1980).

As a quick, constructive way to address the problem of self-gravitating accretion disks, we have recently argued (Bertin 1997) that the energy equations, when self-gravity is dominant, should be replaced by the physically based prescription that $Q$ is self-regulated to a value $\bar{Q}$ close 
to unity. This is all we need if we are interested in calculating the main dynamical characteristics of such selfregulated accretion disks. On the other hand, a detailed examination of the physical justification at the basis of the self-regulation process (see Sect. 3 of the article by Bertin \& Lodato 1999) readily shows that self-regulation results from heating and cooling mechanisms that go beyond those considered in Sect. 2. In the self-regulation picture, widely considered in the study of spiral galaxies, the Jeans instability has the effect of inducing a fast heating of the disk (on the dynamical time-scale), at a rate that depends strongly on $Q$. Such heating thus tends to bring $Q$ towards values of stability. On the other hand, if the system is Jeans-stable to begin with, efficient cooling mechanisms (that, in some cases, may be just the radiative cooling of Eq. (2)) can cool the disk down to lower values of $Q$. In the following sections we will reformulate the energy balance equations starting from the arguments just presented. Such reformulation of the energy equations will allow us to: $(i)$ demonstrate how self-regulation can be established; ( $i i)$ rediscuss the opacity properties in the self-gravitating regime. In addition, it will give us (iii) a starting point for the study of the emission properties of self-gravitating accretion disks; (iv) a tool to analyze the structure of the transition region, where the disk becomes self-gravitating. A study of the last two points is postponed to a separate investigation.

\subsection{Additional heating}

We suggest that in the expression of the heating rate a term $H_{\mathrm{J}}$ should be added (so that $Q^{+}=D+H_{\mathrm{J}}$ ), strongly dependent on the value of $Q$, which we express in terms of surface quantities as:

$H_{\mathrm{J}}=g(Q) \sigma c^{2} \Omega$.

Here $g(Q)$ is a function that rises quickly when $Q$ drops below $\bar{Q}$ (for example, $g(Q) \sim(\bar{Q} / Q)^{n}$, with $n \gg 1$ ). The form of this heating term is argued on dimensional grounds, given the fact that the collective effects associated with self-gravity are expected to heat the disk on the dynamical time-scale. The general structure of $H_{\mathrm{J}}$ is analogous to that of the heating term introduced in the context of spiral structure in galaxies to demonstrate the timedependent process of self-regulation (see Bertin \& Romeo 1988). The argument is also analogous to that used by Lin \& Pringle (1987) to incorporate the collective effects associated with self-gravity into an effective viscosity. In fact, we could write (if we take $h=c^{2} / \pi G \sigma$ ):

$H_{\mathrm{J}}=\left(\alpha_{\mathrm{J}} c h\right) \sigma\left(r \Omega^{\prime}\right)^{2}$,

with the definition:

$\alpha_{\mathrm{J}}=\frac{g(Q)}{Q} \frac{\Omega \kappa}{\left(r \Omega^{\prime}\right)^{2}}$.

With this device, we see that the term we are introducing mimics the viscous heating for a system with modified viscosity $\alpha \rightarrow \alpha+\alpha_{\mathrm{J}}$. In practice, we think that the two issues, of a modified viscosity in the presence of spiral instabilities and of a heating term associated with Jeansrelated instabilities, should be kept separate. Note that in the context of spiral galaxies the emphasis is reversed, and little attention is paid to the problem of viscosity, although it is well known that spiral instabilities eventually favor mass accretion toward the center. In any case, it should be stressed that the present approach remains heuristic. The issue of energy dissipation requires further clarification (see also Balbus \& Papaloizou 1999).

\subsection{Additional cooling}

By referring to the context of galactic disks, we find another important element that is generally overlooked in the discussion of the energy balance equations for accretion disks.

This is the distinction between thermal speed and effective thermal speed. In practice, the effective thermal speed entering the dynamical equations may be higher than the thermal speed entering the radiation equations. The interstellar medium of galaxy disks is a cloudy medium, for which the thermal speed associated with the temperature of the clouds is much smaller than the velocity dispersion of the cold gas clouds. It is the latter that sets the appropriate effective thermal speed relevant to the dynamics of the disk. When a fluid model of the interstellar medium is adopted, in studies of disk dynamics, the numbers that are used for the equivalent acoustic speed are then those applicable to the turbulent speed characteristic of the cloudy medium. Therefore, one important cooling mechanism is that associated with the inelastic collisions between gas clouds. Mechanisms of this kind may operate also in accretion disks, but they are usually ignored (but see Quataert \& Chiang 2000).

\subsection{Modified energy balance equation}

Because of these considerations, we see that the energy balance equation for a non-advective disk should be written as:

$D+H_{\mathrm{J}} \geq R$

In the inner $\operatorname{disk} Q$ is likely to be much larger than unity: if we drop the caveat about the distinction between thermal speed and effective thermal speed, we see that such an equation would then reduce to that of a standard disk.

\section{Basic equations for steady-state self-gravitating accretion disks close to the transition region}

In the following we will study the problem of selfgravitating accretion disks in a region not far from the transition region from non-self-gravitating to selfgravitating disks (see Fig. 1). 


\subsection{Equations}

The analysis is thus simplified in two respects.

(i) On the one hand, the radial balance of forces for a cool, slowly accreting disk will be described by the Keplerian relation:

$\Omega^{2} \simeq \frac{G M_{\star}}{r^{3}}$

where $M_{\star}$ is the mass of the central object. In Eq. (15) we have neglected the contribution of the disk selfgravity. This approximation is valid for $r \ll r_{\mathrm{s}}=$ $2\left(G M_{\star}\right)(\bar{Q} / 4)^{2}(G \dot{M} / 2 \alpha)^{-2 / 3}$, where $\alpha$ is the viscosity parameter (see Eq. (20) below). For parameters typical of an AGN, previous studies (Bardou et al. 1998) show that the transition from a non-self-gravitating to self-gravitating disk occurs at a radius, which we call $r_{Q}$, that is much smaller than $r_{\mathrm{s}}$, so that, at least for a certain parameter range, the approximation in Eq. (15) is applicable to the inner parts of the disk. The effects associated with the integral relation between the gravitational field generated by the disk $\mathrm{d} \Phi_{\sigma} / \mathrm{d} r$ and $\sigma$ are described in the paper by Bertin \& Lodato (1999).

(ii) On the other hand, the energy balance equation is taken to be (for the definitions of $D$ and $H_{\mathrm{J}}$ see Eqs. (1) and (11)):

$D+H_{\mathrm{J}}=R$

with $R$ specified by the relevant radiation mechanism. In the following we will use the expression for the cooling rate per unit volume appropriate for optically thin bremsstrahlung (but see the additional discussion of Sect. 5.3):

$q_{\mathrm{br}}=\sqrt{\frac{2 \pi k T}{3 m_{\mathrm{e}}}} \frac{2^{4} \mathrm{e}^{6}}{3 \hbar m_{\mathrm{e}} c^{3}} \bar{g} n^{2} \simeq 1.410^{-27} T^{1 / 2} \bar{g} n^{2}$,

where $n$ is the particle density, given by $n=\rho / \mu m_{\mathrm{p}}$ ( $\mu$ is the mean molecular weight), and $\bar{g} \approx 1.2$ is a gaunt factor averaged over frequencies (see Rybicki \& Lightman 1979), and where the last expression is evaluated in CGS units ( $T$ measured in K). In Eq. (17) $c$ is the speed of light and should not be confused with the effective thermal speed defined above; we have assumed that the atomic weight is unity, and that $n_{\mathrm{i}}=n_{\mathrm{e}}$. In the spirit of the thin disk approximation, we will define $R=q_{\mathrm{br}}(z=0) h$. The optically thin description breaks down at small radii, below a scalelength $r_{T}$, where the disk is optically thick (see discussion in Sect. 5).

The remaining equations are the equation of continuity:

$\dot{M}=-2 \pi r u \sigma$,

the conservation of angular momentum:

$\dot{J}=\dot{M} r^{2} \Omega+2 \pi \nu \sigma r^{3} \frac{\mathrm{d} \Omega}{\mathrm{d} r}$,

and the standard $\alpha$-prescription (Shakura \& Sunyaev 1973) for viscosity:

$\nu=\alpha c h$, where $h$ is the vertical thickness of the disk, defined by:

$h=\frac{c^{2}}{\pi G \sigma} \frac{\pi}{4 Q^{2}}\left[\sqrt{1+8 Q^{2} / \pi}-1\right]$.

The last equation is an interpolation formula for the "exact" thickness calculated including both the self-gravity of the disk and the gravitational force of the central object in the vertical hydrostatic equilibrium (see Eq. (A9) in the article by Bertin \& Lodato 1999 and discussion below), in the limit of the Keplerian rotation curve. Note that in the limit of a non-self-gravitating disk $(Q \gg 1)$ this equation leads to the commonly used result $h=\sqrt{\pi / 2}(c / \Omega)$. In the following we will take $\dot{J}=0$; the effects of a non-zero net angular momentum flux modify the disk structure only in the innermost parts of the disk.

The level of viscosity present, as measured by the parameter $\alpha$, should depend also on the development of spiral instabilities, and, therefore, on the parameter $Q$. Since for the self-gravitating part of the disk $Q$ will turn out to be self-regulated, we prefer to simplify the analysis by leaving $\alpha$ as an assigned parameter. Our reference value (often considered for AGN-type accretion disks) will be $\alpha=0.05$.

\subsection{Characterization of the transition region}

The above set of equations is naturally reduced to a dimensionless form by the introduction of the length-scale:

$r_{\mathrm{R}}=\frac{4}{\mu m_{\mathrm{p}} m_{\mathrm{e}}}\left[\sqrt{\frac{\pi}{3}} \bar{g} \frac{\mathrm{e}^{6}}{\hbar c^{3}} \frac{4}{9 \alpha}\left(\frac{G \dot{M}}{3 \alpha}\right)^{-1 / 3} \frac{\left(G M_{\star}\right)^{1 / 2}}{2 \pi G}\right]^{2 / 3}$

In practice, this length-scale roughly corresponds to the outermost radius for which our solution exists (see Sect. 5.1 below). In the following, we will thus refer to the dimensionless radius $\hat{r}=r / r_{\mathrm{R}}$.

Based on the scale lengths introduced so far, and on general results that will be better justified in Sect. 5, we can characterize the structure of the accretion disk, in the region we are focusing on, by the following four zones (see Fig. 1): (i) An inner standard disk with the value of $Q$ decreasing rapidly outwards; (ii) A transition zone, in which the heating term due to self-gravity becomes important and the optical thickness of the disk decreases until (iii) the optically thin regime is reached; $(i v)$ At radii of the order of $r_{\mathrm{s}}$ self-gravity also affects the rotation of the disk and at even larger radii the simple set of equations adopted in this section breaks down and possibly other cooling mechanisms come into play.

\subsection{Additional remarks on the role of self-gravity in the region considered}

The term "self-gravitating" is often used with different meanings, because the quantity $\pi G \sigma$ may be judged to be important in relation to different quantities: (a) The cumulative effect of $\pi G \sigma$ may make the gravitational force in 


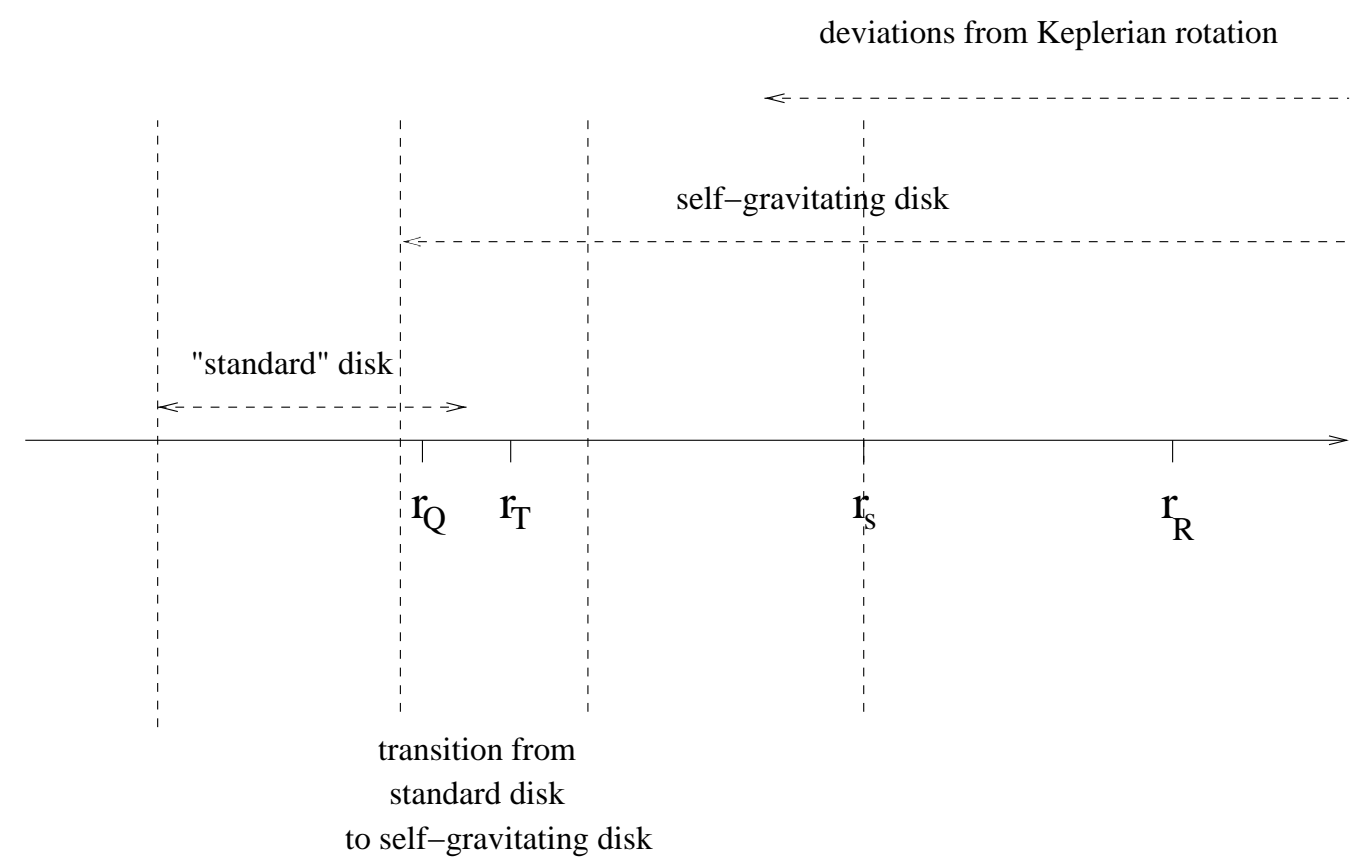

Fig. 1. Sketch of the disk structure and of the different zones with the corresponding scale lengths. In practice, the present paper addresses the third zone, between $r_{T}$ and $r_{\mathrm{s}}$

the plane different from the Keplerian field generated by the central point mass; in this respect, to check whether the disk should be considered to be self-gravitating, we should compare the location $r$ of interest with the scale $r_{\mathrm{s}}$ of the problem. In the limit $r \gg r_{\mathrm{s}}$ the gravitational field of the central point mass may be neglected, but at $r \approx r_{\mathrm{s}}$ the disk is already self-gravitating. (b) The transport properties in the disk may be affected significantly by density waves and other Jeans-related phenomena; here the importance of the disk self-gravity is quantified by comparing $\pi G \sigma$ to the acceleration $c \kappa$ and thus it is quantified by $Q$. Note that, in this respect, for a disk to be selfgravitating it is sufficient that $Q$ be close to unity; in other words, the self-gravitating limit is not set by the condition $Q \ll 1$, as might be thought at first. (c) The vertical scale of the disk may be significantly changed with respect to that of a non-gravitating disk; as shown in a separate article (Bertin \& Lodato 1999; see Fig. A1 and Eq. (A9) in the Appendix), this depends both on the local value of $Q$ and on the overall mass distribution in the disk. Furthermore, the expression of the disk thickness given in Eq. (A9) of Bertin \& Lodato (1999) is valid uniformly and can be considered as an "exact" formula; the self-gravitating expression $h=c^{2} / \pi G \sigma$ is applicable, for example, when $Q \approx 1$ and the gravitational field in the disk plane is dominated by the contribution of a disk characterized by $\sigma \sim 1 / r$.

To a large extent, the three items above are independent aspects of the role of self-gravity, even though we may argue that at very small radii any accretion disk is likely to be non-self-gravitating from all the three points of view, while a cold, radially extended accretion disk at large radii is bound to be self-gravitating with respect to all aspects (a), (b), and (c).
In this paper we are less interested in the specific impact of item (a) (investigated earlier by us) and wish to focus on item (b), which had not been properly explored in relation to the energy equations. Fortunately, effects related to Jeans instability, which we model by means of the heating term described by Eq. (11), become important at a location $r \approx r_{Q}$, which, for models applicable to AGN disks, occurs further inwards with respect to the location where item (a) comes into play, because $r_{Q} \ll r_{\mathrm{s}}$. As regards disk thickness, we recall that the expression given in Eq. (A9) of Bertin \& Lodato (1999) can be considered as an "exact" formula; thus its Keplerian limit, as adopted in Eq. (21), is legitimate when item (a) is unimportant. Therefore, in the transition region, we can safely follow the set of equations described in Sect. 4.1.

\section{Thermal stability of the self-regulated solution}

Optically thin, non-advective disks have been generally discarded because of the thermal instability that results from an increase of the heating rate relative to the cooling rate in response to an increase of the temperature. This occurs because they are found to violate the condition for thermal stability:

$\frac{\mathrm{d}}{\mathrm{d} T}\left(\log \left(\frac{Q^{-}}{Q^{+}}\right)\right)_{\sigma}>0$,

where the subscript $\sigma$ indicates that the derivative should be taken at constant surface density (see, for example, Pringle 1981).

Since our discussion of Sect. 3 modifies the basic ingredients of the energy balance equations, we should now check the properties of the self-gravitating solutions in relation to the thermal stability condition. Given the fact 


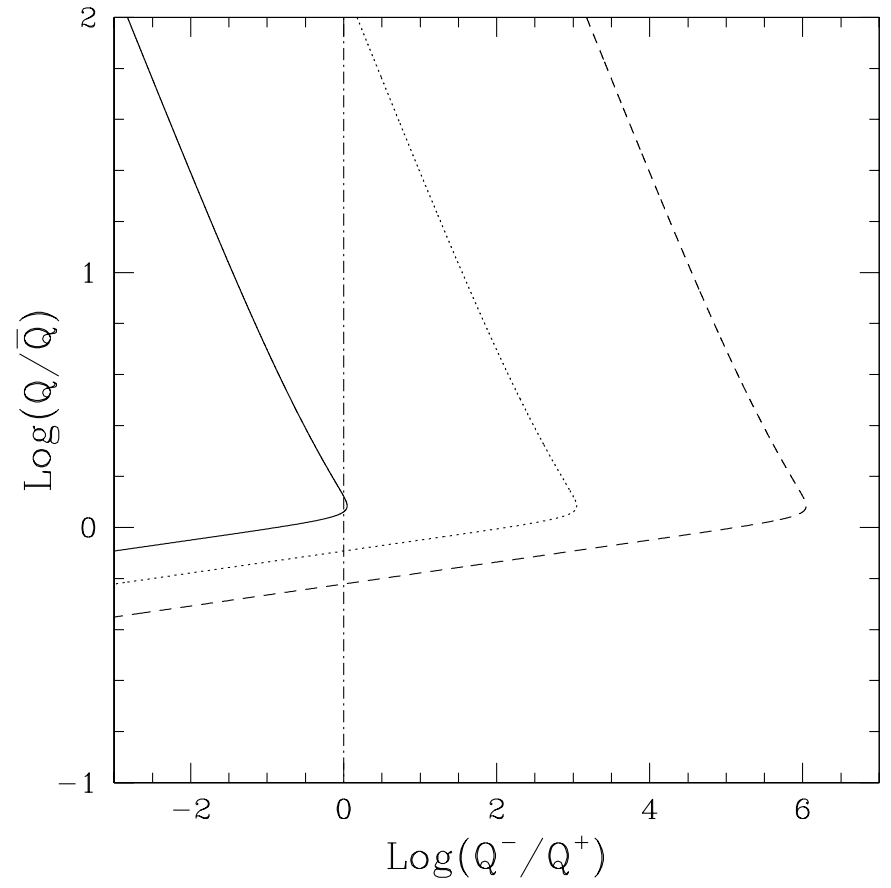

Fig. 2. Relation between the ratio of heating to cooling rates and the stability parameter $Q$ at three different radii, i.e., from right to left $\hat{r}=10^{-4}, 10^{-2}, 1$. The stable branch is the one with positive slope. The intersection between the vertical line and the stable branch is the stable self-regulated solution

that the additional heating term $H_{\mathrm{J}}$ given in Eq. (11) is a function of $Q$, which is proportional to the thermal speed $c$, we can reformulate the thermal stability condition as:

$\frac{\mathrm{d}}{\mathrm{d} Q}\left(\log \left(\frac{Q^{-}}{Q^{+}}\right)\right)_{\sigma}>0$.

\subsection{The thermally stable self-gravitating branch}

In Fig. 2 we show the relation between $Q^{-} / Q^{+}$and $Q$ at fixed radius, for different values of the radius. Equilibrium corresponds to $\log \left(Q^{-} / Q^{+}\right)=0$ (the vertical line in Fig. 2) and the solution is thermally stable if the slope of the curve is positive. As is easily recognized, there are two branches of solutions: one, unstable, is characterized by $Q \gg 1$, for which the dominant heating mechanism is viscous dissipation, as in non-self-gravitating disks (this solution can be regarded as analogous to the SLE solution). The other solution is self-gravitating (it has $Q \approx 1$ ) and it is, indeed, thermally stable. Note that the stable equilibrium solution is always characterized by a value of $Q$ close to unity. The equilibrium $Q$ increases slightly with radius (although this dependence is weak); independent of the physical parameters of the disk, the maximum value of $Q$ for the stable solution is $\approx 1.3 \bar{Q}$. There is a maximum radius for which this kind of solution is possible, located near $r_{\mathrm{R}}$. The relative location of the two scale lengths $r_{\mathrm{R}}$ and $r_{\mathrm{s}}$ defines the importance of the deviation from Keplerian rotation in our model. For parameters typical of AGN configurations $\left(M_{\star}=10^{8} M_{\odot}, \dot{M}=1 M_{\odot} / \mathrm{yr} \approx 610^{25} \mathrm{~g} / \mathrm{s}\right)$,

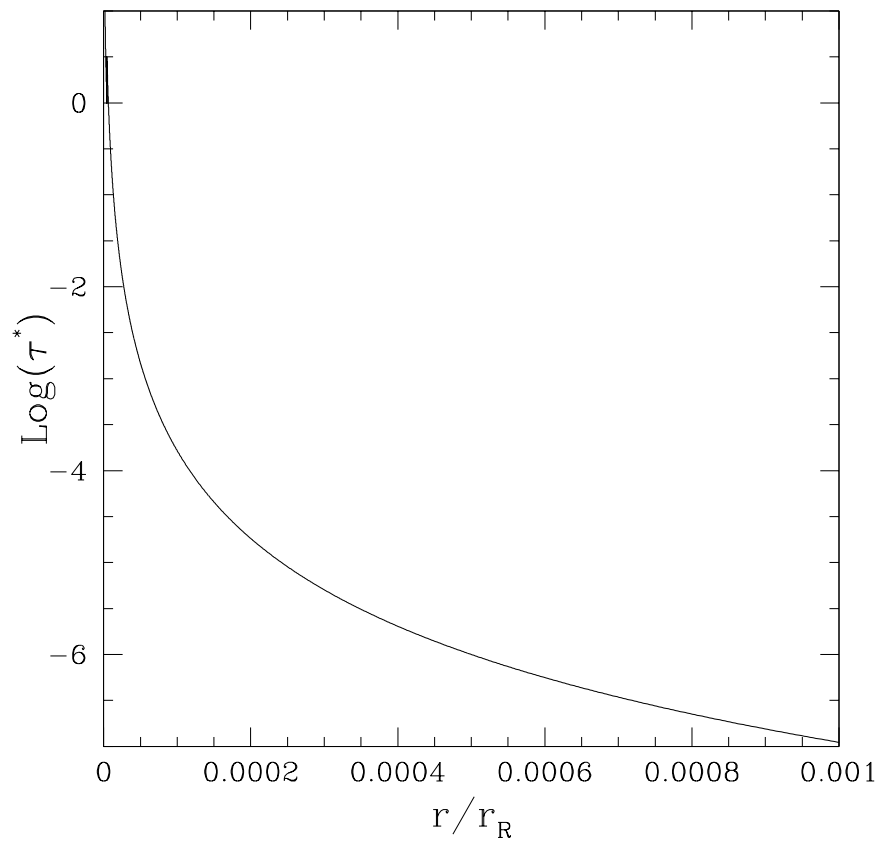

Fig. 3. Optical thickness of the disk as a function of radius for $\dot{M}=1 M_{\odot} /$ yr. In the innermost regions the disk is optically thick

we estimate $r_{\mathrm{R}} / r_{\mathrm{s}} \approx 510^{2}$. Hence, before the scale $r_{\mathrm{R}}$ is reached, the rotation curve is expected to depart from the simple Keplerian curve, so that the quantitative results of the present simplified work are only approximate when $\hat{r}>210^{-3}$ and may even be qualitatively incorrect when $\hat{r}$ is of order of unity. The lack of solutions at large radii $(\hat{r}>1)$ is due to the fact that radiative cooling is insufficient and unable to balance the heating rate. In this context, one should keep in mind that, as we indicated in Sect. 3.2, other kinds of effective cooling could be efficient and play a role in the outer regions, such as inelastic collisions between clouds, which have been neglected here.

\subsection{Consistency of the optically thin description}

We have also calculated the optical depth of the disk, to check that the model of the optically thin disk is consistent. We express the "effective" optical depth $\tau^{*}$ (Rybicki \& Lightman 1979) for the case $\kappa_{T} \gg \kappa_{\mathrm{br}}$ (where $\kappa_{T}$ and $\kappa_{\text {br }}$ are the opacity due to Thomson scattering and bremsstrahlung, respectively), as:

$\tau^{*}=\sqrt{\tau_{\mathrm{br}} \tau_{T}}$

where $\tau_{\mathrm{br}}=\kappa_{\mathrm{br}} \sigma$ and $\tau_{T}=\kappa_{T} \sigma$.

In Fig. 3 we show the optical depth as a function of radius for the thermally stable equilibrium solution. The disk is optically thin in the outer regions and the optical depth increases inwards. In the innermost regions the disk eventually becomes optically thick. This occurs at a dimensionless radius $\hat{r}_{T}$ independent of the central mass and very weakly dependent on the mass accretion rate (approximately $\hat{r}_{T} \propto \dot{M}^{-1 / 18}$ ); for $\dot{M}=1 M_{\odot} /$ yr, the transition to the optically thick region occurs at an inner 


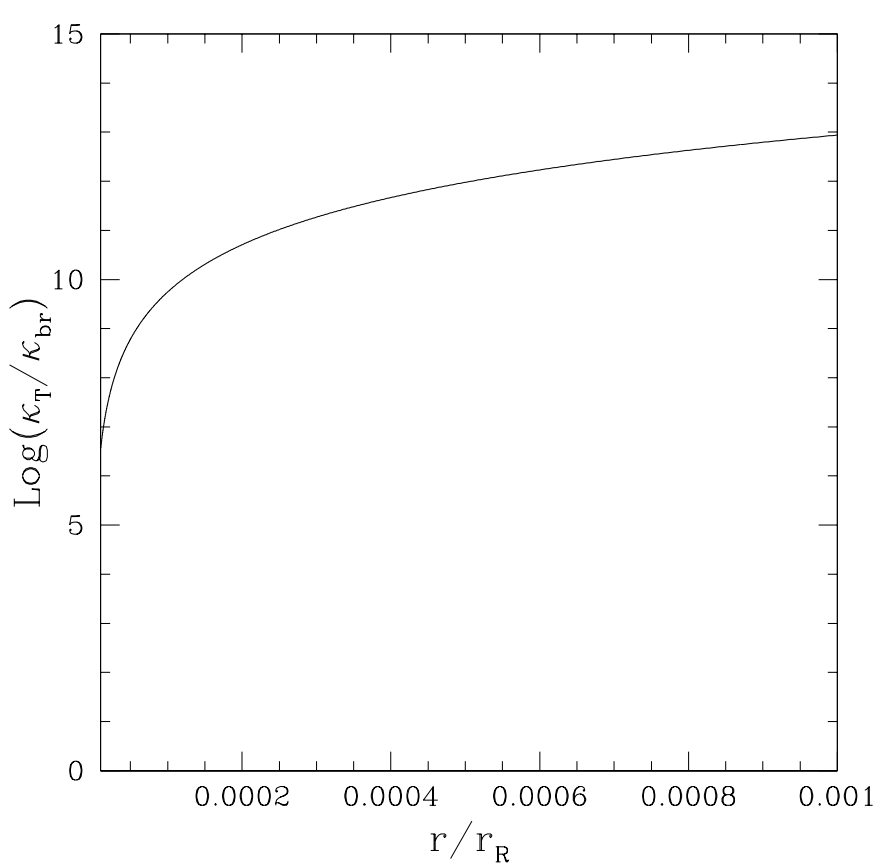

Fig. 4. Ratio of Thomson opacity to free-free opacity, showing that Thomson scattering always dominates over bremsstrahlung

radius $\hat{r}_{T}=r_{T} / r_{\mathrm{R}} \approx 410^{-6}$. In Fig. 4 we show the profile of the ratio $\kappa_{T} / \kappa_{\mathrm{br}}$ to check that the Thomson opacity always dominates over free-free absorption.

In the transition region from optically thick to optically thin emission, for a proper matching, a more detailed description is desired.

\subsection{Robustness with respect to changes in the radiative processes}

In contrast with other cases for which optically thin emission is considered (i.e., the inner regions of disks with very low mass accretion rates), in our case the temperature of the disk (for reasonable values of $\dot{M}$ ) is expected to be low, so that pure bremsstrahlung fails to be the dominant radiative process and line emission and absorption become important. In view of this, we have checked the consequences of a different cooling function, using a functional interpolation (Sarazin \& White 1987) of numerical data (Raymond et al. 1976), appropriate for lower temperatures.

In this test of robustness, the value of the accretion rate $\dot{M}$, through the temperature of the outer disk defined as $k T_{\text {out }}=\left(\mu m_{\mathrm{p}} / 2\right)(G \dot{M} / 3 \alpha)^{2 / 3}$, appears explicitly in the problem. This is a result of the fact that the various line emissions become efficient at specified temperatures. In practice, only minor differences are found for the selfgravitating branch (even under extreme conditions; see Fig. 5), with respect to the previous analysis, because the cooling function influences only the equilibrium value of $Q$ but this is, in any case, self-regulated to values close to unity (on the thermally stable equilibrium solution).

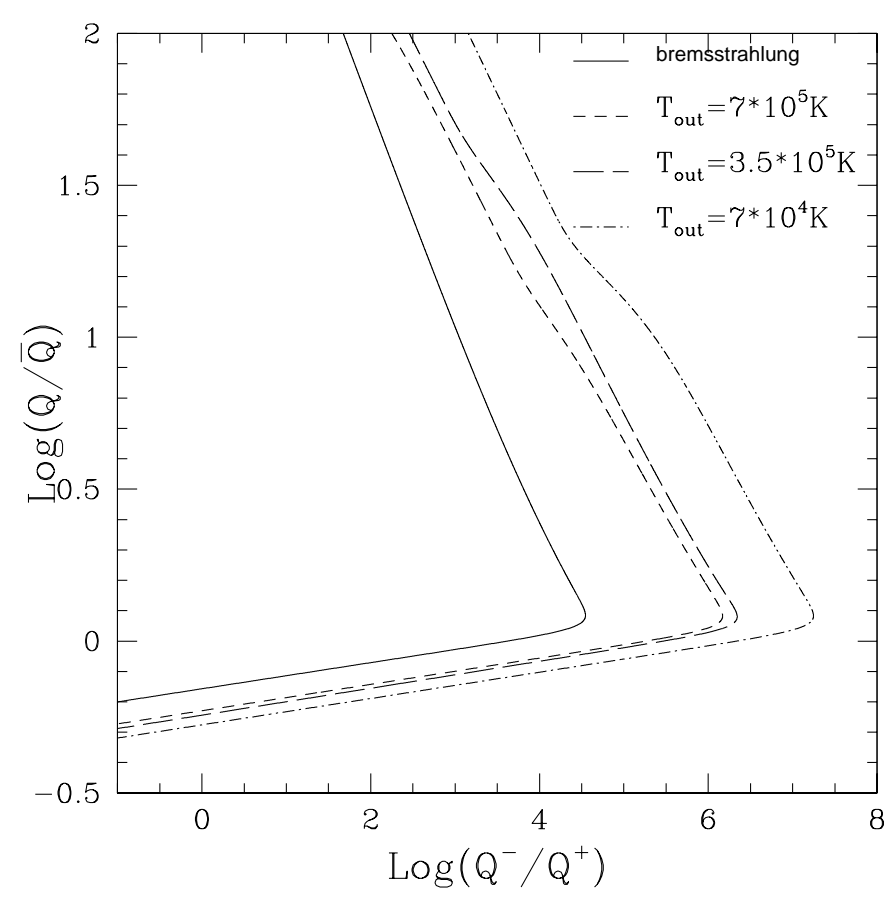

Fig. 5. Stability curves for different choices of the cooling function at radius $\hat{r}=10^{-3}$. The details of the cooling mechanism do not change the main characteristics of on the stable selfgravitating branch of solutions. The three outer temperatures correspond to $\dot{M} \simeq 90,30,3 M_{\odot} /$ yr, respectively. Here the value $\alpha=0.05$ has been assumed

\section{Discussion and conclusions}

Self-gravitating accretion disks may be present in many astrophysical systems, ranging from protostellar disks to AGN and could also be of interest for the general galactic context.

In protostellar disks it is likely that, especially at the beginning of the accretion processes, during which the mass of the central object is small, self-gravity plays an important role. The influence of self-gravity has sometimes been invoked to explain the flat infrared spectrum of some T Tau stars (Adams et al. 1987; Duschl et al. 2000). However, current estimates for the mass accretion rates in these systems (typically $\dot{M} \sim 10^{-8} M_{\odot} / \mathrm{yr}$ ) so far have discouraged interpretations of the shape of the spectrum in terms of self-gravitating, optically-thick disks.

On the other hand, observational evidence suggests that, in some cases, the currently accepted models may be inadequate to describe the accretion flow of some AGN. For example, in the case of the Seyfert galaxy NGC 1068, deviations from Keplerian rotation have been observed; when the standard Shakura \& Sunyaev (1973) model is applied to this case, the values of the stability parameter $Q$ in the outer disk turn out to be exceedingly low (Kumar 1999). In fact, the energy balance in standard disks, for which viscous dissipation is exactly balanced by radiative heating, leads to a $Q$ profile decreasing rapidly outwards.

In this paper, we have focused on the problem of the energy budget in self-gravitating accretion disks, with the ultimate goal of providing a framework to 
describe the emission and the spectrum associated with such disks. We have adopted the view that the effects of self-gravity on the energy balance are not only related to viscous dissipation, but that there are also processes, such as the self-regulation of Jeans instability, that should give a significant contribution to the heating of the disk, at least in its outer parts.

By referring to conditions applicable to some AGN, we have then shown that a thermally stable, non-advective, optically thin equilibrium solution is possible in the region dominated by self-gravity. In contrast with other optically thin solutions (Shapiro et al. 1976), our solution is thermally stable as a result of the strong dependence of the heating term related to self-gravity on the parameter $Q$. We have also found an unstable solution, which is not selfgravitating $(Q \gg 1)$, and we argue that it is a modification of the SLE solution.

The value of $Q$ on the thermally stable solution is always close to unity, and it is slightly dependent on radius. The temperature in the outer disk is constant and depends on the mass accretion rate $\dot{M}$. The structure of the disk, as long as the energy balance is dominated by selfgravity, turns out to depend only weakly on the detailed radiation processes involved. This has been shown by taking into account two different radiation processes: pure bremsstrahlung and the case of a cooling function dominated by line absorption and emission (Sarazin \& White 1987), which is best suited for the low temperature conditions expected in the outer disk. The dependence on the radiation processes is only weak because of the selfregulation mechanism: whatever the radiation process, the disk readjusts, so as to keep $Q$ close to marginal stability.

For simplicity, in this paper we have focused on conditions relevant to the region of the disk where the transition to the self-gravitating regime occurs. Further out, one should include the presence of additional cooling mechanisms, not considered here, and especially the effects related to the deviations from a Keplerian rotation curve. The latter effects have been widely discussed in a previous paper (Bertin \& Lodato 1999).

\section{References}

Adams, F. C., Lada, C. J., \& Shu, F. H. 1987, ApJ, 312, 788

Balbus, S. A., \& Papaloizou, J. C. B. 1999, ApJ, 521, 650

Bardou, A., Heyvaerts, J., \& Duschl, W. J. 1998, A\&A, 337, 966

Bertin, G. 1997, ApJ, 478, L71

Bertin, G., \& Romeo, A. B. 1988, A\&A, 195, 105

Bertin, G., \& Lodato, G. 1999, A\&A, 350, 694

Duschl, W. J., Strittmatter, P. A., \& Biermann, P. L. 2000, A\&A, 357, 1123

Kolykhalov, P. I., \& Sunyaev, R. A. 1980, Sov. Astron. Lett., 6, 357

Kumar, P. 1999, ApJ, 519, 599

Laughlin, G., \& Bodenheimer, P. 1994, ApJ, 436, 335

Lin, D. N. C., \& Pringle, J. E. 1987, MNRAS, 225, 607

Lin, D. N. C., \& Pringle, J. E. 1990, ApJ, 358, 515

Meyer, F., \& Meyer-Hofmeister, E. 1981, A\&A, 104, L10

Narayan, R., \& Popham, R. 1993, Nature, 362, 820

Narayan, R., \& Yi, I. 1994, ApJ, 428, L13

Narayan, R., \& Yi, I. 1995, ApJ, 452, 710

Paczyński, B. 1978, Acta Astron., 28, 91

Piran, T. 1978, ApJ, 221, 652

Pringle, J. 1981, ARA\&A, 19, 137

Quataert, E., \& Chiang, E. I. 2000, ApJ, 543, 432

Raymond, J. C., Cox, D. P., \& Smith, B. W. 1976, ApJ, 204, 290

Rybicki, G. B., \& Lightman, A. P. 1979, Radiative Processes in Astrophysics (Wiley \& Sons, New York)

Sarazin, C. L., \& White, R. E. 1987, ApJ, 320, 32

Shakura, N. J., \& Sunyaev, R. A. 1973, A\&A, 24, 337

Shapiro, S. L., Lightman, A. P., \& Eardley, D. M. 1976, ApJ, 204, 187 (SLE)

Yuan, F. 1999, ApJ, 521, L55 